\title{
COMBINATORIAL PROPERTIES FOR BLACKWELL SETS
}

\author{
R. M. SHORTT
}

(Communicated by Thomas J. Jech)

\begin{abstract}
Under the assumption of $\mathrm{CH}$ (continuum hypothesis) we produce a strongly Blackwell set whose product with a standard space and whose intersection with an analytic set are not Blackwell. Previously, such examples were known to exist only under Martin's Axiom (MA) and not- $\mathrm{CH}$.
\end{abstract}

0. Introduction. Blackwell sets, which function in the category of measurable spaces as an analogon to compact spaces in the topological category, have been the focus of some recent papers $[\mathbf{1 - 5}, \mathbf{8}]$. For the motives behind such study, as well as a survey of basic results, the reader is referred to [1]. In particular, Jakub Jasinski [5] has shown that, with the assumption of Martin's Axiom (MA) and the negation of the Continuum Hypothesis $(\mathrm{CH})$, these spaces are poorly behaved under the operations of product and intersection.

By making use of some theory developed with the assistance of Bhaskara Rao [8], it is possible to demostrate similar pathologies under the assumption of $\mathrm{CH}$. A construction is given in the proposition of $\S 3$ infra.

1. Preliminaries. A measurable space $(X, B)$ is separable if its Borel structure $B=B(X)$ is countably generated (c.g.) and contains all singleton sets drawn from $X$. If $Y \subset X$, then $(Y, B(Y))$ is again separable, where $B(Y)=\{B \cap Y: B \in B(X)\}$. A aeparable space $(S, B)$ is standard if there is a complete separable metrizable (i.e., Polish) topology on $S$ whose Borel $\sigma$-field is $B=B(S)$. A subset of a separable spaces is analytic if it is a measurable image of a standard space. Any two uncountable standard spaces are Borel-isomorphic [6, pp. 487-489].

A separable space $X$ has the Blackwell property if $B(X)$ does not properly contain any c.g. sub- $\sigma$-algebra separating points of $X$. A separable space $X$ has the strong Blackwell property if any two c.g. sub- $\sigma$-algebras of $B(X)$ with the same atoms coincide. Every analytic space has the strong Blackwell property. For proof of this and other facts concerning Blackwell sets, consult the monograph [1].

Let $S$ be a standard space. A subclass $I$ of $B(S)$ is a $\sigma$-ideal if it is closed under countable unions and has the property that $N \cap B \in I$ whenever $N \in I$ and $B \in B(S)$. If $N \subset S$, we write $\langle N\rangle$ to denote the subset $\langle N\rangle=(N \times S) \cup(S \times N)$ of $S \times S$. A subset $R$ of $S \times S$ is I-reticulate if $R \subset\langle N\rangle$ for some $N \in I$. A subset $X$ of $S$ is $I$-dense in $S$ if $X$ has nonvoid intersection with every set in $B(S) \backslash I$. A set $X \subset S$ is I-dense of order 2 in $S$ if $X \times X$ meets every set $R \in B(S \times S)$ which

Received by the editors September 20, 1986.

1980 Mathematics Subject Classification (1985 Revision). Primary 54H05; Secondary 28A05, $03 \mathrm{E} 15$

Key words and phrases. Blackwell spaces, analytic sets, sigma-ideals.

Research supported by National Science Foundation grant DMS-8412413. 
is not I-reticulate. Let $T \subset S \times S$ be the graph of a Borel-isomorphism between two sets in $B(S)$. The set $T$ is an I-thread if it is not $I$-reticulate.

A subset $X$ of $S$ is $I$-Lusin if $X$ is uncountable and $X \cap N$ is countable for each $N \in I$. If $m$ is a Borel probability on a Polish space $S$, and $I$ is the $\sigma$-ideal of $m$-measure zero (resp. first category) sets in $B(S)$, then the $I$-Lusin sets are commonly called Sierpiński (resp. Lusin) sets. For further explication of the idea, see [7].

These notions enter into the study of Blackwell properties by dint of the following results.

LEMMA 1. Let $S$ be a standard space and let I be a $\sigma$-ideal in $B(S)$. If $X$ is an I-Lusin set I-dense of order 2 in $S$, then $X$ is strongly Blackwell.

INDICATION. Use Lemma 2 and Proposition 1 in [8].

LEMMA 2. Let $S$ be a standard space and let I be a $\sigma$-ideal in $B(S)$. If $X \subset S$ is a Blackwell set which is I-dense in $S$, then $X \times X$ intersects every I-thread in $S \times S$.

INDICATION. See the proof of Proposition 1 in [8].

These results greatly facilitate the construction (and destruction) of sets with Blackwell properties. The following is an example which will be used later.

LEMMA 3. Suppose that $X$ is a subset of a standard space $S$ such that $X \times S$ is Blackwell. Then for each analytic set $A \subset(S \times S) \backslash(X \times X)$ there is an analytic set $Q \subset S$ such that $A \subset\langle Q\rangle$ and such that $Q \cap X$ is empty.

Proof. Let $I$ be the $\sigma$-ideal of all sets $N \in B(S \times S)$ such that $N \cap(X \times S)$ is empty. Clearly, $X \times S$ is $I$-dense in $S \times S$. Since $A$ is analytic, there is some measurable surjection $f: S \rightarrow A$. Define a Borel subset $T$ of $(S \times S) \times(S \times S)$ by

$$
T=\left\{\left(s_{1}, s_{2}, t_{1}, t_{2}\right): s_{2}=t_{2} \text { and } f\left(s_{2}\right)=\left(s_{1}, t_{1}\right)\right\} .
$$

Then $T$ is disjoint from $(X \times S) \times(X \times S)$. Furthermore, each section of $T$ (in either direction) over any point of $S \times S$ is at most a singleton. Now Lemma 2 implies that $(X \times S) \times(X \times S)$ meets every I-thread in $(S \times S) \times(S \times S)$. It must be, therefore, that $T$ is I-reticulate. So there is a set $N \in I$ with $T \subset\langle N\rangle$. Let $p: S \times S \rightarrow S$ be the natural projection to the first coordinate. Then $Q=p(N)$ is an analytic subset of $S$ such that $A \subset\langle Q\rangle$ and such that $Q \cap X$ is empty. Q.E.D.

2. Main results. We investigate the preservation of Blackwell properties under the operations of product and intersection. It seems that not much can be said in general. Jasinski [5] has shown that under the assumption of MA and not-CH, various pathologies surface. We complete the picture by exhibiting such behaviors under $\mathrm{CH}$. But first, two examples of regular conduct are examined.

LEMMA 4. Let $X$ be a subset of a standard space $S$ and let $A \subset S$ be an analytic set. If $X \times S$ is strong Blackwell, then so are $X \times A$ and $X \cap A$.

Proof. The space $X \times A$ is a measurable image of $X \times S$ and so is also strong Blackwell [1, p. 24]. Now consider the diagonal $D=\{(s, s): s \in S\}$ in $S \times S$. Since $D \cap(X \times A)$ is isomorphic with $X \cap A$, it follows that this set is strong Blackwell [1, p. 27]. Q.E.D. 
Say that a subset $X$ of a standard space $S$ is analytically separated if for each analytic set $A \subset S$ with $X \cap A=\varnothing$ there is a set $N \in B(S)$ with $A \subset N$ and $X \cap N=\varnothing$. Such sets include all analytic sets, all Sierpiński sets, and all Lusin sets [7].

LEMMA 5. Suppose that $X$ is an analytically separated subset of an uncountable standard space. Let $A$ be an uncountable analytic set. The following are equivalent:

(1) $X \times S$ is Blackwell;

(2) $X \times S$ is strong Blackwell;

(3) $X \times A$ is Blackwell;

(4) $X \times A$ is strong Blackwell.

ProOF. The implication $(2) \rightarrow(4)$ follows from Lemma 4, whilst $(4) \rightarrow(3)$ is obvious. Since $A$ contains an uncountable standard set [6, p. 444], $X \times S$ is isomorphic with a Borel subset of $X \times A$. So (3) $\rightarrow(1)$.

To show $(1) \rightarrow(2)$, we employ Lemmas 1 and 3 . Let $I$ be the $\sigma$-ideal in $B(S \times S)$ comprising sets whose intersection with $X \times S$ is countable. Clearly, $X \times S$ is $I$ Lusin. We shall prove that $X \times S$ is $I$-dense of order 2 in $S \times S$. Lemma 1 will then imply that $X \times S$ is strong Blackwell.

Given a Borel set $R \subset(S \times S) \times(S \times S)$ not intersecting $(X \times S) \times(X \times S)$, define $p:(S \times S) \times(S \times S) \rightarrow S \times S$ to be the projection map $p\left(s_{1}, s_{2}, s_{3}, s_{4}\right)=\left(s_{1}, s_{3}\right)$. Then $p(R)$ is an analytic subset of $(S \times S) \backslash(X \times X)$. By Lemma 3 , there is an analytic set $Q \subset S$ such that $p(R) \subset Q$ and such that $Q \cap X$ is empty. It will follow from the separation property for $X$ that there is some $N \in B(S)$ with $N \cap X=\varnothing$ and such that $p(R) \subset\langle N\rangle$. Then $R \subset\langle N \times S\rangle$, and $(N \times S) \cap(X \times S)=\varnothing$. Thus $X \times S$ is $I$-dense of order 2. Q.E.D.

PROPOSITION $(\mathrm{CH})$. Let $S$ be an uncountable standard space. Then there is a strong Blackwell subset $X$ of $S$ and an analytic subset $A \subset S$ such that neither $X \times S$ nor $X \cap A$ is Blackwell.

DEMONSTRATion. We realize $S$ as the union of intervals $(-1,0) \cup(0,1)$ under the usual linear structure. Let $f: S \rightarrow S$ be the Borel automorphism defined by $f(s)=-s$. Let $A^{+}$be an analytic, non-Borel subset of $(0,1)$ and put $A=$ $A^{+} \cup f\left(A^{+}\right)$. Let $A_{0}$ be the graph of the restriction of $f$ to the set $A$.

Now define $I$ to be the $\sigma$-ideal in $B(S)$ generated by all standard subsets of $A$ and all standard subsets of $S \backslash A$. Each set in $I$ decomposes into the union of two such sets. Let $R_{0} R_{1} R_{2} \cdots R_{\alpha} \cdots, \alpha<c$, be a transfinite listing of all sets in $B(S \times S)$ that are not $I$-reticulate. Call a subset $P$ of $A$ trivial if there is some $N \in I$ with $P \subset N$. List in transfinite series $P_{0} P_{1} \cdots P_{\alpha} \cdots, \alpha<c$, all nontrivial analytic subsets of $A$. Also list the sets in $I$ as $N_{0} N_{1} \cdots N_{\alpha} \cdots, \alpha<c$, and define $M_{\alpha}=\bigcup\left\{N_{\beta}: \beta \leq \alpha\right\}$.

Observe that if $R$ is a set in $B(S \times S)$ which is not $I$-reticulate, then $R$ is not a subset of $A_{0}$. Choose a point $\left(x_{0}, y_{0}\right)$ in $R_{0} \backslash\left(\left\langle M_{0}\right\rangle \cup A_{0}\right)$. Define $U_{0}=\left\{x_{0}, y_{0}\right\} \cap A$ and select a point $z_{0}$ from $P_{0} \backslash f\left(U_{0}\right)$. Put $X_{0}=\left\{x_{0}, y_{0}, z_{0}\right\}$.

In general, we assume that for each $\beta<\alpha$, a countable set $X_{\beta}$ has been defined so that $\left(X_{\beta} \times X_{\beta}\right) \cap R_{\beta}$ and $X_{\beta} \cap P_{\beta}$ are nonvoid, while $\left(X_{\beta} \times X_{\beta}\right) \cap A_{0}=\varnothing$. Also, we assume that for $\gamma \leq \beta \leq \alpha$ one has $X_{\beta} \cap M_{\gamma}=X_{\gamma} \cap M_{\gamma}$. Define $Y_{\alpha}=\bigcup\left\{X_{\beta}: \beta<\alpha\right\}$ and $Z_{\alpha}=Y_{\alpha} \cap A$. 
Noting the assumption of the continuum hypothesis, we select a point $\left(x_{\alpha}, y_{\alpha}\right)$ from $R_{\alpha} \backslash\left(\left\langle M_{\alpha} \cup f\left(Z_{\alpha}\right)\right\rangle \cup A_{0}\right)$. Define $U_{\alpha}=Z_{\alpha} \cup\left(\left\{x_{\alpha}, y_{\alpha}\right\} \cap A\right)$ and select an element $z_{\alpha}$ from $P_{\alpha} \backslash f\left(U_{\alpha}\right)$. Put $X_{\alpha}=Y_{\alpha} \cup\left\{x_{\alpha}, y_{\alpha}, z_{\alpha}\right\}$. Then $\left(X_{\alpha} \times X_{\alpha}\right) \cap R_{\alpha}$ and $X_{\alpha} \cap P_{\alpha}$ are nonnull, whilst $\left(X_{\alpha} \times X_{\alpha}\right) \cap A_{0}=\varnothing$. Also, if $\gamma \leq \alpha$, then $X_{\alpha} \cap M_{\gamma}=X_{\gamma} \cap M_{\gamma}$.

Define $X=\bigcup\left\{X_{\alpha}: \alpha<c\right\}=\left\{x_{\alpha}, y_{\alpha}, z_{\alpha}: \alpha<c\right\}$. Clearly, $X$ is I-Lusin and $I$-dense of order 2 in $S$. It follows from Lemma 1 that $X$ has the strong Blackwell property.

Now note that $(X \times X) \cap A_{0}=\varnothing$. Suppose that $Q$ is an analytic subset of $S$ with $A_{0} \subset\langle Q\rangle$. Then put $P=Q \cap A$ and observe that $A_{0} \subset\langle P\rangle$.

ClaIm 1. The analytic set $P$ is nontrivial. Suppose that this is not the case. Then $P \subset N \subset A$ for some $N \in B(S)$. Thus $A_{0} \subset\langle P\rangle \subset\langle N\rangle$. It follows that $A_{0}=A_{0} \cap\langle N\rangle=\operatorname{graph}(f) \cap\langle N\rangle$ is a Borel set in $S \times S$, as is its one-one projection $A$. This contradiction establishes the claim.

ClaIm 2. If $P \subset A$ is a nontrivial analytic set, then $X \cap P$ is uncountable. If, in such an instance, $X \cap P$ is countable, then $P \backslash X$ is another nontrivial analytic set. But $X$ meets every such set. The contradiction yields the claim.

From Claims 1 and 2 it follows that whenever $Q$ is an analytic subset of $S$ with $A_{0} \subset\langle Q\rangle$, then $X \cap Q$ is uncountable. Using Lemma 3, we see that $X \times S$ is not Blackwell.

We now show that $X \cap A$ is not a Blackwell set. First, note that $X \cap A$ is I-dense in $S$. This is because $B \cap A$ is not trivial if $B$ is a Borel subset of $S$ not in $I$. Second, we see that $\operatorname{graph}(f)$ is not $I$-reticulate in $S \times S$ : use the argument in Claim 2 supra. Finally, the construction of $X$ shows that, $[(X \cap A) \times(X \cap A)] \cap \operatorname{graph}(f)=$ $(X \times X) \cap A_{0}=\varnothing$.

Lemma 2 now implies that $X \cap A$ is not a Blackwell set. Q.E.D.

NOTE. The continuum hypothesis was needed to ensure the I-Lusin property of $X$. Since every analytic (or coanalytic) set is the union of $\aleph_{1}$ Borel sets [6, p. 483], this assumption appears to be essential.

3. Open questions. Can one produce (under any consistent axioms) a subset $X$ of a standard space $S$ such that $X \times S$ is Blackwell, but not strongly Blackwell? Examples of Blackwell, not strongly Blackwell sets $X$ have been constructed under $\mathrm{MA}$ and not- $\mathrm{CH}[\mathbf{3}-\mathbf{5}]$ and under $\mathrm{CH}[\mathbf{4}, \mathbf{8}]$.

Can one obtain a Blackwell set $X$ and an analytic set $A$ such that $X \cup A$ is not Blackwell? This has been accomplished under MA and not- $\mathrm{CH}$ [5], but the situation in ZFC or even ZFC and $\mathrm{CH}$ is not known.

Is the main proposition true in $\mathrm{ZFC}$ ?

\section{REFERENCES}

1. K. P. S. Bhaskara Rao and B. V. Rao, Borel spaces, Dissertationes Math. 190 (1981), 63 pp.

2. W. Bzyl, On analytic-dense Blackwell sets, Colloq. Math. (to appear).

3. W. Bzyl and J. Jasinski, A note on Blackwell spaces, Bull. Polish Acad. Sci. 31 (1983), 215217.

4. D. Fremlin, On Blackwell algebras (preprint).

5. J. Jasinski, On the combinatorial properties of Blackwell spaces, Proc. Amer. Math. Soc. 93 (1985), 657-660.

6. K. Kuratowski, Topology, vol. I, Academic Press, New York; PWN, Warsaw, 1966. 
7. A. W. Miller, Special subsets of the real line, Handbook of Set-theoretic Topology, Elsevier/ North-Holland, Amsterdam, 1984.

8. R. M. Shortt and K. P. S. Bhaskara Rao, Generalised Lusin sets with the Blackwell property, Fund. Math. (to appear).

Department of Mathematics, Wesleyan University, Middletown, ConnectiCUT 06457 